\title{
Nanostructure Analysis of Sol-gel PZT Thin Films Derived from Different Chemical Routes
}

Aiying Wu, P. M. Vilarinho

Department of Ceramics and Glass Engineering, CICECO, University of Aveiro, Aveiro, Portugal aiying@ua.pt

Lead zirconate - lead titanate (PZT) materials are commercially important piezoelectric and ferroelectrics in a wide range of applications, such as data storage (dynamic access and ferroelectric random access memories) and sensing and actuating devices. PZT with the morphotropic phase boundary composition offers the highest piezoelectric response and at the present there are no fullydeveloped alternative materials to PZT. The importance of PZT associated with the continuous requirements of device miniaturization, imposes the development of high quality PZT thin films with optimized properties. Concomitantly due to the dependence of the final properties of thin films on the details of the microstructure a thoroughly analysis at the local scale of their microstructure is necessary. Sol-gel method, is one of the Chemical Solution Deposition techniques used to prepare oxide thin films, such as PZT. Starting from a solution, a solid network is progressively formed via inorganic polymerisation reactions. Most metal alkoxides used for sol-gel synthesis are highly reactive towards hydrolysis and condensation. Therefore their chemical reactivity has to be tailored via the chemical modification (or complexation) of metal alkoxides to avoid uncontrolled reactions and precipitation. For PZT sol gel thin film preparation, two chemical routes are frequently used depending on the nature of the molecular precursor, namely methotoxyethanol (MOE) route and diol-route. In a MOE route, the precursors, lead acetate trihydrate, titanium isopropoxide and zirconium propoxide, are modified with MOE to change their reactivity. In a diol route, dihydroxy alcohol, such as ethylene glycol or propanediol, is used as a solvent to modify the metal alkoxides. Though XRD pattern of the films derived from different routes showed perovskite structure, these films displayed different final morphology, microstructure and dielectric properties. In the current work a detailed analysis of the local structure of PZT films synthesised from these two routes by means of scanning electron microscopy (SEM) and atomic force microstructure (AFM), is conducted. Local electric properties via piezo-force microscopy (PFM) are also analyzed and correlated to their microstructures.

Figure 1 shows the surface morphology obtained by atomic force microscopy of PZT films derived from diol $(1 \mathrm{a}-1 \mathrm{c})$ and MOE (1d-1f). The RMS (root mean square) surface roughness of PZT films derived from diol route is relatively smaller $(2.62 \mathrm{~nm})$ than the roughness of the films derived from MOE route $(8.12 \mathrm{~nm})$. At the same time these last films possess a bimodal grain size distribution easily identified from the deflection image (Fig. 1e), in which big grains are indicated by a green arrow and a group of small grains specified by a red arrow. In accordance, topography of surface analysis (1c, 1f) evidences a single modal distribution of grain height for diol derived films and a multimodal distribution for MOE derived ones.

Local piezo-response has been studied in detail on individual domains of the films and Figure 2 depicts their local piezoelectric properties. Local piezoloops of MOE derived PZT films (Fig. 2b) clearly show a dependence on the grain size: for grains with similar domain orientation, big grains show a higher average remanent $d_{33}$ value $(\sim 32)$ than the small grains with lower $d_{33}$ value $(\sim 28)$. In 
comparison the piezo-response signal of different grains in diol derived PZT films showed similar $\mathrm{d}_{33}$ values. Domains with opposite orientation (black domain and white domain) have been tested and the measured $\mathrm{d}_{33}$-loops are similar. In conclusion: $\mathrm{i}$ ) the microstructure of PZT films as a function of processing route has been clearly visualized by AFM and details at the nano-scale electrical response related with the microstructure undoubtedly highlighted by PFM and ii) because the macroscopic electric response of these films results from the average reaction of domains switching under the electric field that by itself depend on the individual response of each domain, the knowledge of the individual domain behaviour is fundamental to understand and optimise the dielectric properties of PZT and other ferroelectric thin films.

Financial support from CICECO, FCT and FEDER is acknowledged.
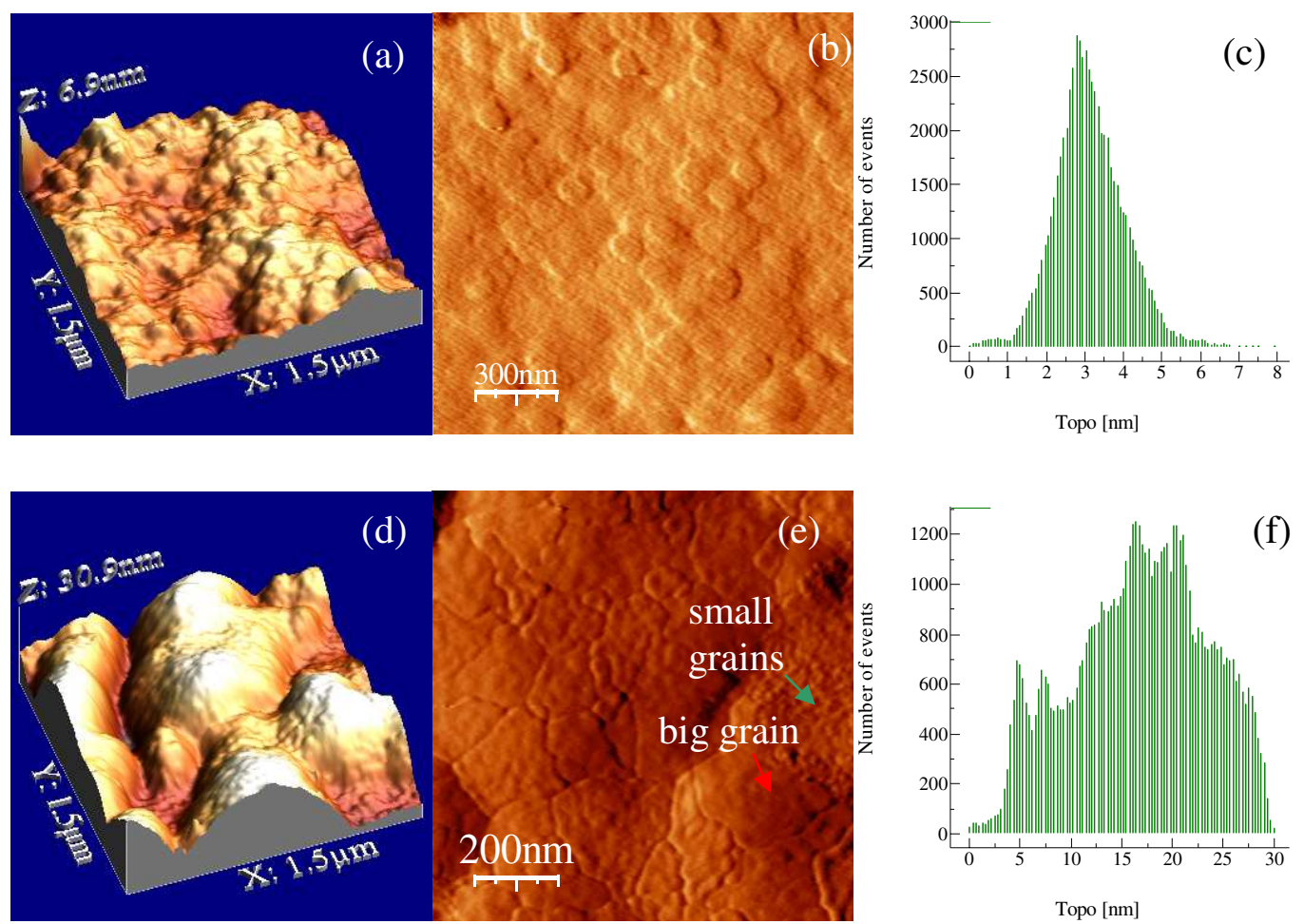

Figure 1 Microstructure of PZT films revealed by AFM: surface morphology of PZT films derived with diol $(a-c)$ and MOE (d-f).
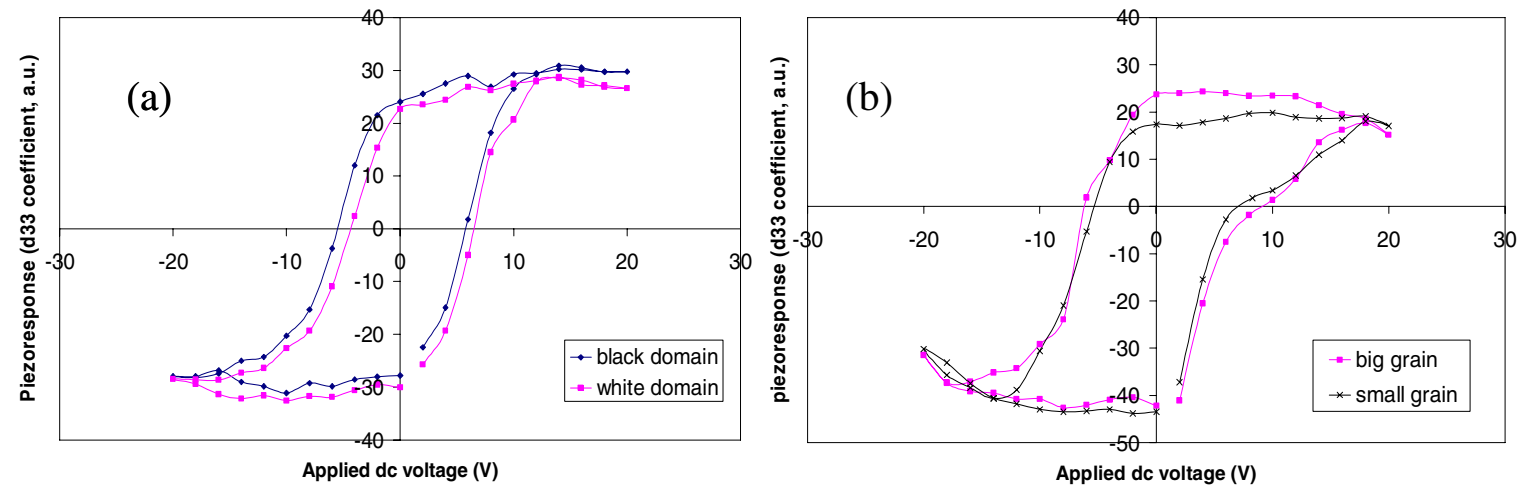

Figure 2 Local piezoelectric hysteresis-loops of PZT films derived from different routes: (a) Diolderived and (b) MOE derived film. 